\title{
ST-segment elevation myocardial infarction after COVID-19 reinfection: The disseminated thrombotic process
}

\author{
Tomasz A. Michalski*, Tomasz Figatowski*, Milosz J. Jaguszewski \\ $1^{\text {st }}$ Department of Cardiology, Medical University of Gdansk, Poland
}

This paper was guest edited by Prof. Marek Koziński

\begin{abstract}
A 64-year-old man with no medical history of coronary events was admitted due to a diagnosis of myocardial infarction with prominent ST-segment elevation. He suffered from coronavirus disease 2019 (COVID-19) 7 weeks prior (with a subsequent negative test) as well as arterial hypertension, type 2 diabetes, and obesity. Upon admission, the PCR SARS-CoV-2 screening test was positive anew. Coronary angiography showed a thrombus in the left main (LM) coronary artery with $90 \%$ stenosis (Fig. 1A-D). Distal segments of the left anterior descending (LAD) artery and diagonal branch (Dg) were occluded by thromboembolic material. After an intracoronary bolus of eptifibatide, thrombectomy, and balloon angioplasty in LAD, the operator decided to proceed with drug-eluting stent implantation (Fig. 1E, F). In LM, an intervention was completed without residual stenosis with Throm-
\end{abstract}

bolysis in Myocardial Infarction (TIMI) 3. The distal segment of LAD and Dg remained TIMI 1 and 0 , respectively. Thoracic computed tomography demonstrated specific pulmonary changes (Fig. $1 \mathrm{G}$ ). An echocardiogram showed a left ventricle (LV) ejection fraction of $44 \%$ and a thrombus in the LV apex (Fig. 1H, I; Suppl. Video 1). Notably, there was no atrial fibrillation in the past. On discharge, the patient was prescribed warfarin (international normalized ratio 2.0-2.5) with acetylsalicylic acid, and ticagrelor for 3 and 12 months, respectively. A 3-month follow-up revealed no recurrence of COVID-19 and cardiovascular events. The control echocardiography showed dissolution of the LV thrombus; therefore, warfarin therapy was discontinued.

Herein, we present an elegant case reflecting the potential need for an aggressive antithrombotic treatment during and after COVID-19.

Conflict of interest: None declared

Address for correspondence: Tomasz A. Michalski, MD, $1^{\text {st }}$ Department of Cardiology, Medical University of Gdansk, ul. Dębinki 7, 80-952 Gdańsk, Poland, tel: +48 500359592, e-mail: tomasz.michalski190@gmail.com

Received: 17.06.2021 Accepted: 9.08.2021

*Contributed equally

This article is available in open access under Creative Common Attribution-Non-Commercial-No Derivatives 4.0 International (CC BY-NC-ND 4.0) license, allowing to download articles and share them with others as long as they credit the authors and the publisher, but without permission to change them in any way or use them commercially. 


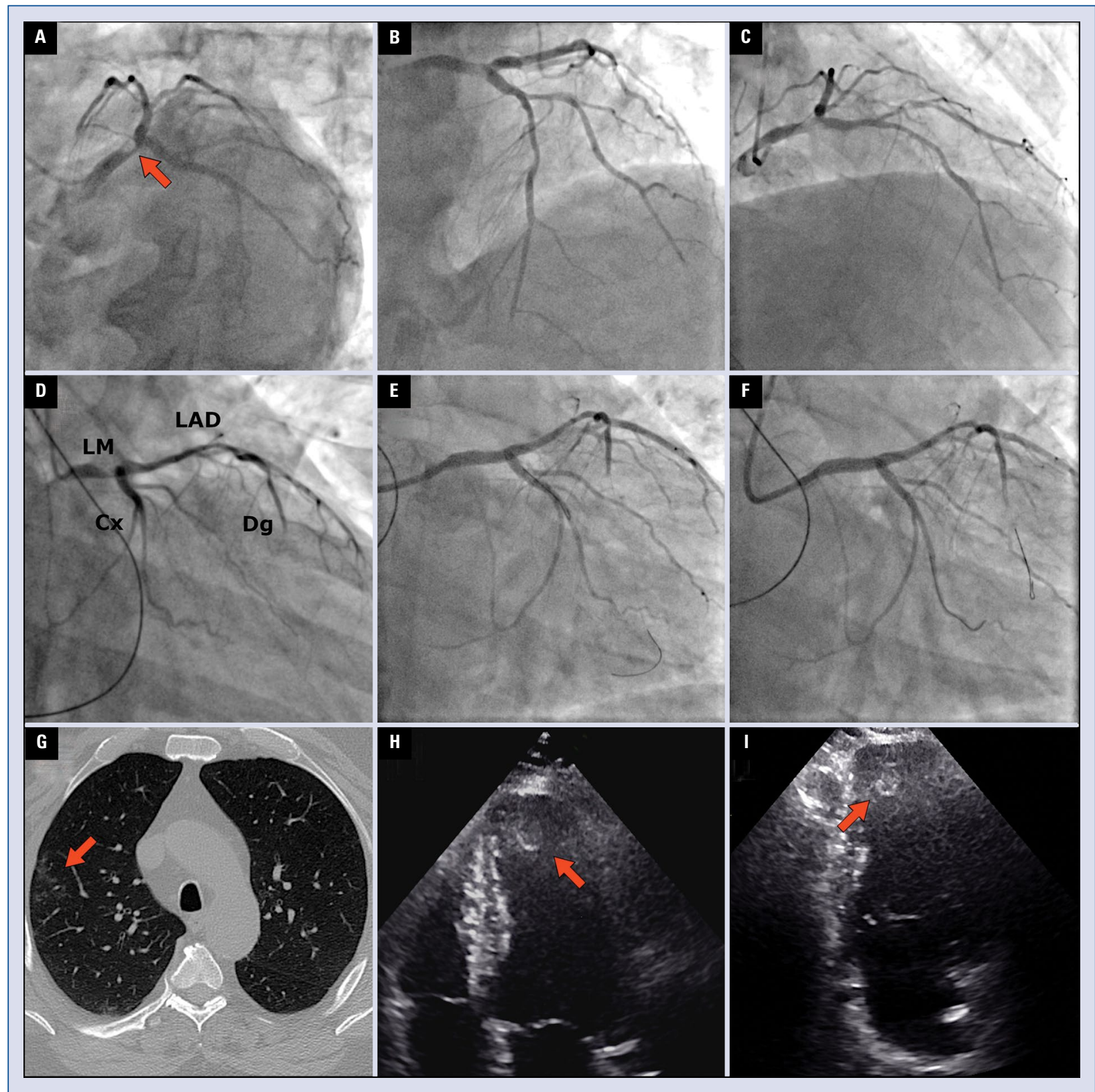

Figure 1. A-D. Thrombus in the left main coronary artery (LM; arrow) and occlusion of the distal left anterior descending artery (LAD) and diagonal branch (Dg); E. Direct stenting technique in the LM; F. Proximal optimization of the stent in the LM; G. Specific for COVID-19 interstitial post-inflammatory changes (arrow) in the lungs; $\mathbf{H}, \mathbf{I}$. Thrombus $(17 \mathrm{~mm} \times 10 \mathrm{~mm}$; arrow) in the apex of the left ventricle visualized in both apical 4- and 2-chamber view of transthoracic echocardiography; $\mathrm{Cx}$ - circumflex artery. 